\title{
Taurocholate Induces Connective Tissue Growth Factor Expression in Hepatocytes Through ERK-YAP Signaling
}

\author{
Bin Yu Guan-nan Jina,b Mei Mac Hui-fang Liang ${ }^{a} \quad$ Bi-xiang Zhang ${ }^{a}$ \\ Xiao-ping Chen ${ }^{\mathrm{a}} \quad$ Ze-yang Ding ${ }^{\mathrm{a}}$
}

aHepatic Surgery Center, Tongji Hospital, Tongji Medical College, Huazhong University of Science and Technology, Wuhan, Hubei, 'bepartment of Nephrology, Union Hospital, Tongji Medical College, Huazhong University of Science and Technology, Wuhan, Hubei, 'Department of Pathophysiology, Key Lab of Neurological Disorder of Education Ministry, School of Basic Medicine, Tongji Medical College, Huazhong University of Science and Technology, Wuhan, China

\section{Key Words}

Cholestasis • Liver fibrosis - Bile acid • Connective tissue growth factor • Extracellular signalregulated kinase $\cdot$ Yes-associated protein

\begin{abstract}
Background/Aims: Cholestasis is characterized by intrahepatic accumulation of cytotoxic bile acids (BAs), ultimately leading to fibrosis and cirrhosis, but the precise role of BAs in cholestasis-induced liver fibrosis remains largely elusive. In this study, we investigated the role and the potential mechanisms of BAs during cholestasis in vivo and in vitro. Methods: The effect of BAs during cholestasis was studied in bile duct ligation (BDL) rat models in vivo. We performed immunohistochemistry, Western blotting, and quantitative RT-PCR to investigate the expression of connective tissue growth factor (CTGF/CCN2) in rat liver during cholestasis. The hepatic cell lines AML12 and BRL were stimulated with taurocholate (TC) and the level of CTGF/CCN2, and activation of ERK, Akt, p38 MAPK, JNK, YAP, and TGF- $\beta / S m a d$ signaling were examined using Western blotting. Next, to elucidate the mechanism underlying bile acidinduced CTGF/CCN2, we treated the cells with MEK1/2 inhibitor (U0126), YAP function inhibitor (verteporfin), p38 kinase inhibitor (SB203580), Akt inhibitor (MK2206), and small interfering RNA (siRNA) targeting mek1, erk, and yap in cooperation with TC. Besides, we confirmed the activation of these signaling pathways in $\mathrm{BDL}$ and sham rat livers by immunohistochemistry, Western blotting, and quantitative RT-PCR. Results: In this study, we confirmed that the expression of CTGF/CCN2 was increased in BDL-induced rodent cholestatic liver fibrosis. In addition, we showed that TC, the main component of BAs, enhanced the synthesis of CTGF/ CCN2 in AML12 and BRL hepatic cell lines. Moreover, we demonstrated that TC activated ERK, Akt, and YAP signaling in hepatocytes, but the precise roles of these signaling cascades in the expression of CTGF/CCN2 were different: TC-induced expression of CTGF/CCN2 was mediated by ERK-YAP signaling, whereas Akt signaling inhibited ERK signaling and YAP and

\footnotetext{
Ze-yang Ding

Hepatic Surgery Center, Tongji Hospital, Tongji Medical College;

Huazhong University of Science and Technology, Wuhan (China)

E-Mail zeyangding@tom.com
}

\section{KARGER}




\section{Cellular Physiology Cell Physiol Biochem 2018;50:1711-1725

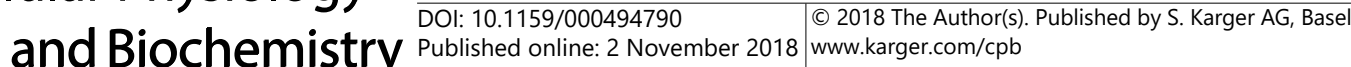 \\ Yu et al.: Taurocholate Induces CTGF in Hepatocytes}

subsequently the expression of CTGF/CCN2 in hepatocytes. Furthermore, YAP functioned as a downstream regulator of ERK signaling in TC-induced CTGF/CCN2 expression in hepatocytes. Conclusion: Our report provides evidence for the role of conjugated BAs in liver fibrosis and suggests that the production of CTGF/CCN2, induced by conjugated BAs via ERK-YAP axis activation, may be a therapeutic target in cholestasis-induced liver fibrosis.

(C) 2018 The Author(s)

Published by S. Karger AG, Basel

\section{Introduction}

Cholestatic liver diseases are characterized by intrahepatic accumulation of bile acids (BAs) irrespective of etiology [1]. Many diseases are accompanied by cholestasis such as primary biliary cholangitis (PBC) [2], primary sclerosing cholangitis (PSC) [3], and biliary atresia [4]. Persistent cholestasis can progress to liver fibrosis and ultimately to endstage liver disease. However, there is limited understanding of the molecular mechanisms underlying cholestasis and cholestasis-related liver fibrosis at present [5].

BAs are the major organic components in the bile and are known as critical mediators in lipid metabolism. In recent investigations, BAs have been found to be important molecules in inflammatory and fibrotic signaling cascades. Under physiological conditions, the concentration of BAs is tightly restricted $[6,7]$, whereas the homeostasis of BA is disrupted during cholestasis. Although there are some adaptative regulatory mechanisms such as inhibition of BA synthesis by fibroblast growth factor 19 (FGF19) [8] and downregulation of the basolateral $\mathrm{Na}^{+}$-dependent bile acid uptake system [9] in the liver, and decreasing the absorption of BA in the ileal [10], the level of BAs in liver tissues is still strongly elevated in human cholestatic liver disease and rodent cholestasis models [11]. Previous studies reported that taurocholate (TC) is one of the most significant elevated BAs during cholestasis $[12,13]$. As the common pathological change in cholestatic disease, high BA concentration is hypothesized to be responsible for the development of cholestatic liver injury [14]. Inflammatory signals are activated by BAs in hepatocytes [15], and BAs can cause apoptosis [16] or necrosis of hepatocytes [17] at different concentrations and induce proliferation of hepatic stellate cells (HSCs) [18], all of which contribute to liver fibrosis. Recently, it has been reported that apart from toxicity, BAs can act as activators of various signaling pathways such as JNK [19], AKT [20], and ERK [21] in the liver.

Connective tissue growth factor (CTGF/CCN2) is implicated in several biological processes like angiogenesis, adhesion, extracellular matrix remodeling, migration, cell survival, and wound healing $[22,23]$; it is considered as a major fibrogenic factor in liver fibrosis [24, 25]. Several cytokines participate in regulating CTGF/CCN2 expression through different signaling pathways. TGF- $\beta$ can induce CTGF/CCN2 expression in a Smaddependent manner in hepatocytes [26], whereas it regulates the expression of CTGF/CCN2 through Smad-independent signaling in hepatic progenitor cells [27]. CTGF/CCN2 also acts as a downstream gene of Yes-associated protein (YAP) [28], which has been reported to be involved in cholestasis $[29,30]$. Moreover, enhanced expression of CTGF/CCN2 was observed in liver tissues of cholestatic patients [31]. However, the relationship between BA accumulation and elevated level of CTGF/CCN2 in the cholestatic liver has not been well explored so far.

In this study, we found that CTGF/CCN2 level was increased in cholestatic liver injury and confirmed that TC induced CTGF/CCN2 expression in hepatocytes. In addition, we found that the ERK-YAP signaling axis was responsible for TC-mediated CTGF/CCN2 expression, whereas TC-activated Akt signaling antagonized ERK-YAP signaling and inhibited the expression of CTGF/ CCN2. 


\section{Cellular Physiology Cell Physiol Biochem 2018;50:1711-1725 \begin{tabular}{ll|l} 
and Biochemistry Published online: 2 November 2018 & $\begin{array}{l}\text { @ } 2018 \text { The Author(s). Published by S. Karger AG, Basel } \\
\text { www.karger.com/cpb }\end{array}$ \\
\hline DOI: 10.1159/000494790
\end{tabular} \\ Yu et al.: Taurocholate Induces CTGF in Hepatocytes}

\section{Materials and Methods}

\section{Cell Lines and Culture}

The hepatic cell line AML12 was kindly provided by Professor Jian-Guo Song (State Key Laboratory of Cell Biology, Innovation Center for Cell Signaling Network, Institute of Biochemistry and Cell Biology of the Shanghai Institutes for Biological Sciences, and Chinese Academy of Sciences) and cultured as described previously [32]. BRL hepatocytes were purchased from China Center for Type Culture Collection (CCTCC, Wuhan, China) and maintained in Dulbecco modified Eagle medium (Gibco, Thermo Fisher Scientific, Shanghai, China) supplemented with $10 \%$ fetal bovine serum (Gibco).

\section{Reagents and Antibodies}

TC and verteporfin (VP) were purchased from Sigma-Aldrich (St. Louis, MO). Cycloheximide and SB203580 were purchased from Beyotime Institute of Biotechnology (Shanghai, China). U0126 and MK2206 were purchased from Cayman Chemicals (Ann Arbor, MI). Antibodies against $\alpha$-smooth muscle actin ( $\alpha$-SMA), YAP, phospho-Akt (Ser473), Akt, phospho-ERK (Thr202/Tyr204), ERK, phospho-p38 (Thr180/Tyr182), p38, phospho-JNK (Thr183/Tyr185), JNK, phospho-Smad2 (Ser465/467), phospho-Smad3 (Ser423/425), phospho-MEK1/2 (Ser221), and $\beta$-actin were purchased from Cell Signaling Technology (Beverly, MA); antibodies against CTGF/CCN2, Smad2/3, and MEK1 were purchased from Santa Cruz Biotechnology Inc.

\section{Animals and Bile Duct Ligation (BDL) Rat Model}

A total of 12 healthy male Sprague-Dawley rats (body weight 190-210 g) were provided by the Experimental Animal Center of Tongji Medical College, Huazhong University of Science and Technology (Wuhan, China) and housed under a 12h light/12h dark cycle with free access to water and normal chow. All animal study protocols were approved by the Committee on the Ethics of Animal Experiments of Tongji Medical College. For the BDL rat model, after anesthesia with ether, the common bile duct was ligated with 5-0 silk ligatures (Ethicon, Johnson \& Johnson, Shanghai, China) and excised; rats in the control group underwent a sham operation. All rats were sacrificed 4 weeks later under ether anesthesia after $12 \mathrm{~h}$ of fasting; liver tissue was obtained and stored for further analysis.

\section{Histological Analysis of Liver Sections}

Formalin-fixed liver tissues were embedded in paraffin and sections were stained with hematoxylin and eosin and Sirius Red (Servicebio Technology Co., Ltd., Wuhan, China) according to the manufacturer's instructions. Immunohistochemical analysis was performed as described previously [27]. Representative images were obtained by Scope-image.

\section{Real-time Quantitative PCR}

Total RNA was extracted using TRIzol reagent (Invitrogen, Carlsbad, CA). Next, $2 \mu \mathrm{g}$ of total RNA was reverse transcribed into cDNA using PrimeScript ${ }^{\circledR}$ RT reagent Kit (Takara, Shanghai, China). Primer sequences are listed in Table 1. Real-Time quantitative PCR was carried out using a CFX96 Touch $^{\text {TM }}$ Real- $^{-}$ Time PCR Detection System (Bio-Rad, Hercules, CA) with SYBR Green PCR Master Mix-PLUS (Toyobo, Osaka, Japan) according to the manufacturer's instructions.

\section{Western Blot Assays}

Tissue and cell lysates were resolved using Sodium dodecyl sulfate-polyacrylamide gel electrophoresis and transferred to polyvinylidene difluoride membranes. After blocking with Tris-buffer saline (TBS) containing $5 \%$ non-fatty milk or bovine serum albumin for $0.5 \mathrm{~h}$ at $37^{\circ} \mathrm{C}$, the membranes were probed with specific primary antibody overnight at $4^{\circ} \mathrm{C}$. Following washing with TBS containing $0.1 \%$ Tween 20 , membranes were incubated with the respective horseradish

Table 1. Primer sequences for quantitative PCR

\begin{tabular}{lccc}
\hline Specie & Genes & Forward sequence $\left(5^{\prime}-3^{\prime}\right)$ & Reverse sequence $\left(5^{\prime}-3^{\prime}\right)$ \\
\hline Rat & GAPDH & GACATCAAGAAGGTGGTG & CAGCATCAAAGGTGGAAG \\
& $\alpha$-SMA & TGTGCTGGACTCTGGAGATG & GAAGGAATAGCCACGCTCAG \\
& CTGF & CGCCAACCGCAAGATTG & ACACGGACCCACCGAAGAC \\
& YAP & CTGGAGCAGGATGGAGGGA & CACGCTGTTCAGGAAGTCGTC \\
& TGF- $\beta$ & CGGACTACTACGCCAAAGAAGT & TGGTTTTGTCATAGATTGCGTT \\
Mouse & GAPDH & CTTTGGCATTGTGGAAGGGC & CAGGGATGATGTTCTGGGCA \\
& CTGF & GGGCCTCTTCTGCGATTTC & ATCCAGGCAAGTGCATTGGTA \\
& YAP & CCACCAGATCTCATTATAGATGG & ACCTAGTAAGTACCAGTTTCCCAGT \\
& TGF- $\beta$ & GCCACTGCCCATCGTCTACT & CACTTGCAGGAGCGCACAAT \\
\hline
\end{tabular}




\section{Cellular Physiology Cell Physiol Biochem 2018;50:1711-1725 \begin{tabular}{ll|l} 
and Biochemistry & $\begin{array}{l}\text { DOl: 10.1159/000494790 } \\
\text { Published online: } 2 \text { November } 2018\end{array}$ & $\begin{array}{l}\text { 2018 The Author(s). Published by S. Karger AG, Basel } \\
\text { www.com/cpb }\end{array}$ \\
\cline { 2 - 3 }
\end{tabular} \\ Yu et al.: Taurocholate Induces CTGF in Hepatocytes}

peroxidase-conjugated secondary antibodies. Immunoreactive bands were detected using ChemiDoc ${ }^{\mathrm{TM}}$ Imaging Systems (Bio-Rad). For each immunoblot, optical density of the bands was measured with AlphaView software (Alphatron Asia Pte. Ltd., Singapore) and normalized to the optical density of the loading control.

Plasmids and Luciferase Reporter Assay

The CTGF-promoter-luciferase gene reporter (CTGF-luc) was constructed as described previously [27]. pRL-TK was purchased from Promega (Madison, WI). Both plasmids were transfected using Lipofectamine 3000 (Life Technologies, Carlsbad, CA) according to the manufacturer's instructions. Luciferase activity was detected as described previously [27].

Transient RNA Interference

Small interfering RNA (siRNA) targeting erk1, erk2, mek1, yap, and scrambled siRNA were designed and synthesized by Ribobio (Guangzhou, China). siRNAs were transfected into the AML12 and BRL cells using Lipofectamine 3000. Efficiency of gene silencing was detected $60 \mathrm{~h}$ after the transfection.

\section{Statistical Analyses}

Data are presented as mean \pm standard error of mean (SEM) from three independent experiments. Statistical analyses were performed by Student's t-test or one-way analysis of variance (ANOVA) using GraphPad Prism 5.0. A value of $\mathrm{p}<0.05$ was considered statistically significant.

\section{Results}

CTGF/CCN2 Is Upregulated in the BDL Rat Model Liver

We first performed BDL in rats to induce cholestatic liver injury, then liver tissue was collected for further analyses. As expected, we detected evidence of cholestasis in the liver and plasma of BDL rats (Fig. 1A). Liver tissue in the BDL group showed inflammatory cell infiltration and bile duct hyperplasia (Fig. 1B). Sirius Red staining indicated increased collagen deposition in cholestatic livers of the BDL group and immunohistochemistry, qRTPCR and Western blotting for $\alpha$-SMA, indicative of hepatic stellate cell (HSC) activation, showed marked elevation in the BDL group (Fig. 1B-D). These findings suggest that BDLinduced cholestasis rats had developed liver fibrosis. Also, immunohistochemistry revealed increased CTGF/CCN2 levels in liver tissue of the BDL rats compared to that of controls, and that CTGF/CCN2 was mainly expressed in the hepatocytes. Consistent with the results of immunohistochemistry, qRT-PCR and Western blot analyses revealed that CTGF/CCN2 was dramatically upregulated in BDL rat liver tissue compared with that in the sham group (Fig. 1C, D). These results suggest that the expression of CTGF/CCN2 is increased in the liver during cholestasis-induced liver fibrosis.

\section{TC induces CTGF/CCN2 expression in hepatocytes}

To determine whether BAs regulate the expression of CTGF/CCN2 in hepatocytes, we used two hepatic cell lines: AML12 and BRL. Under the stimulation of taurocholate (TC), the major elevated conjugated BAs during cholestasis $[12,13]$, CTGF/CCN2 was upregulated in a time- and dose-dependent manner in both AML12 and BRL cell lines (Fig. 2A, B). CTGF/CCN2 mRNA level was consistently increased under TC stimulation (Fig. 2C). Moreover, treatment with cycloheximide blocked TC-induced CTGF/CCN2 expression (Fig. 2D), suggesting that TC promoted CTGF/CCN2 synthesis in vitro. Collectively, these results demonstrate that TC induced CTGF/CCN2 expression in hepatocytes. 


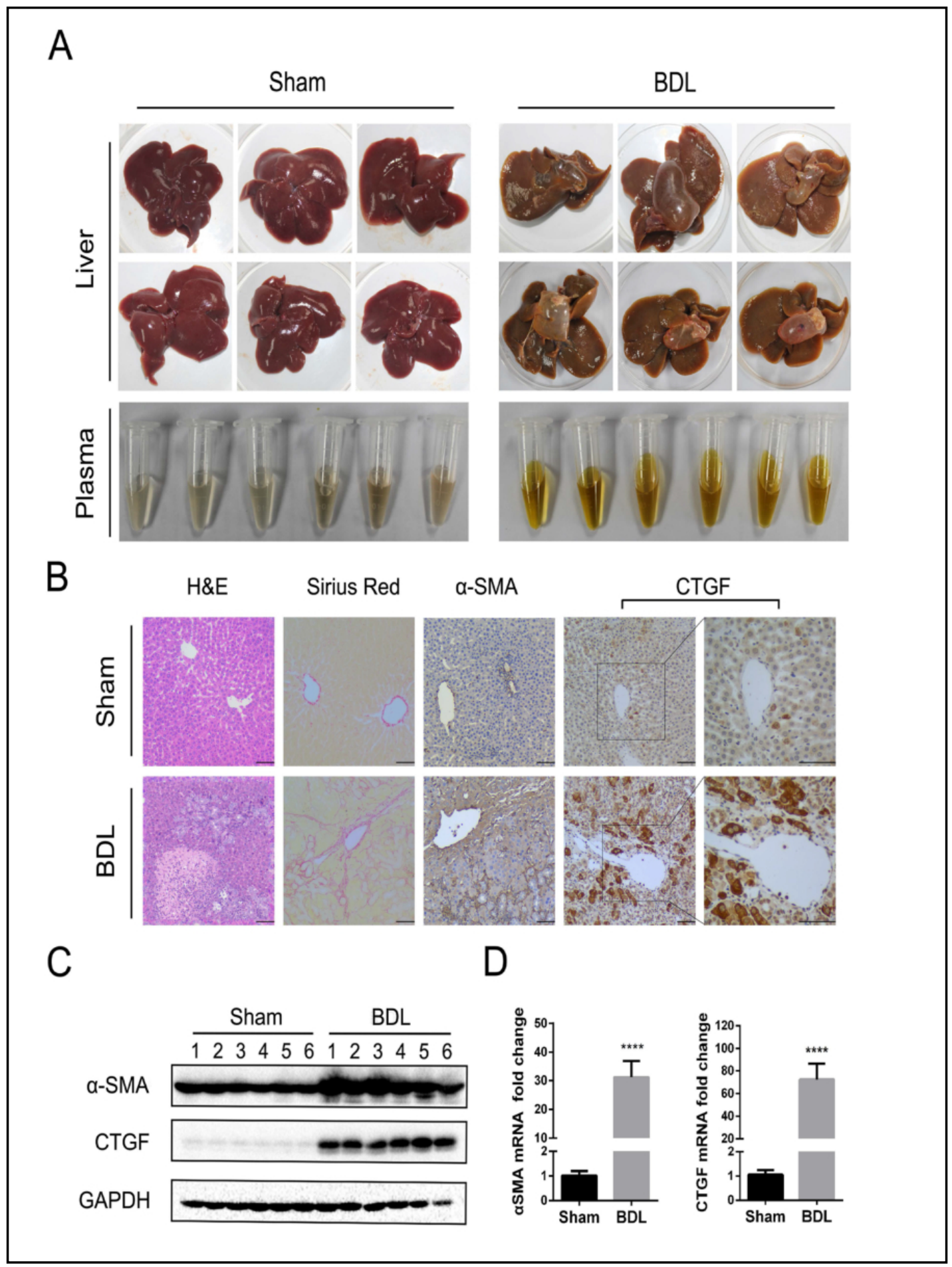

Fig. 1. CTGF/CCN2 is up-regulated in the livers of cholestasis-induced liver fibrosis. (A) The gross appearances of the livers and plasma from BDL and sham rats; (B) Sections of BDL and sham rats liver samples were subjected to H\&E staining, Sirius Red staining and immunohistochemical analysis of $\alpha$-SMA, CTGF/CCN2. Representative images were taken, scale bar, $100 \mu \mathrm{m}$; (C) Lysates of liver tissues from BDL and sham rats were subjected to Western blot analyses with antibodies against $\alpha$-SMA, CTGF/CCN2. GAPDH was used as a loading control; (D) mRNA levels of $\alpha$-SMA, and CTGF/CCN2 of liver tissues from BDL and sham rats were detected by real-time PCR. Relative mRNA levels were normalized to GAPDH and represented as mean \pm S.E.M. $(\mathrm{n}=3)$. ${ }^{* * * *}, \mathrm{p}<0.0001$. 


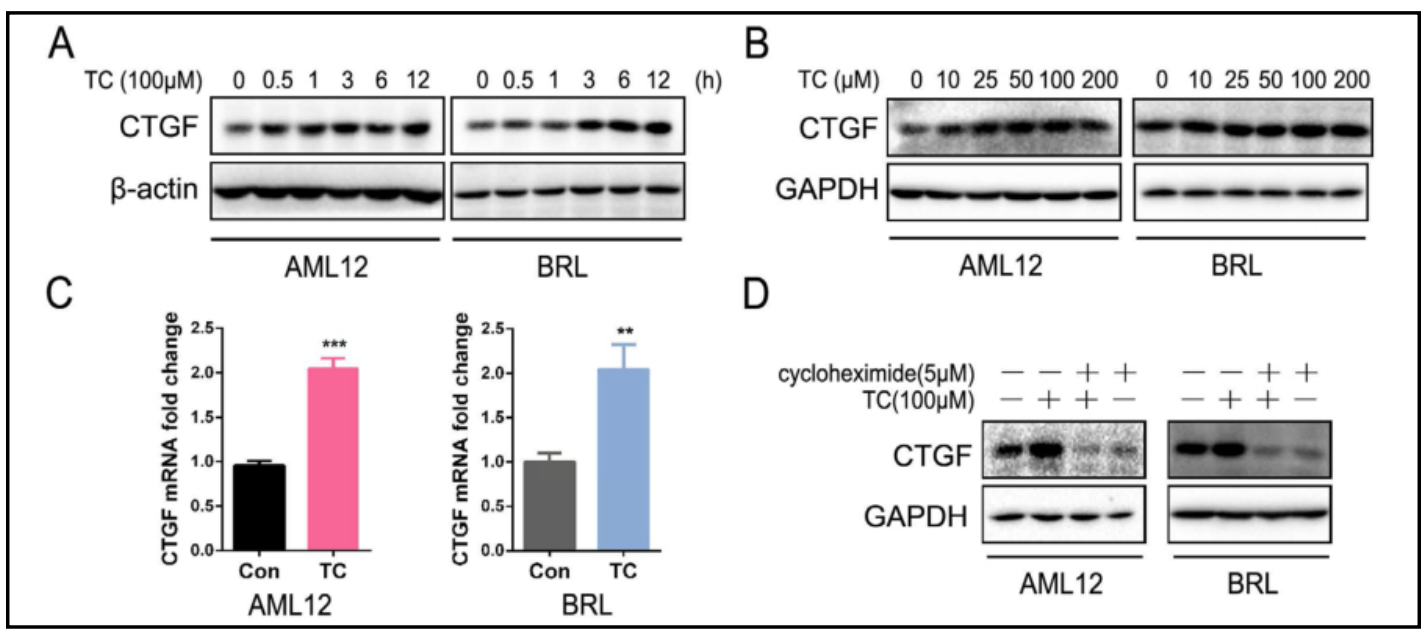

Fig. 2. TC promotes the synthesis of CTGF/CCN2 in hepatocytes.(A) AML12 and BRL cells were treated with TC $(100 \mu \mathrm{M})$ for the indicated times. Lysates were subjected to Western blot analysis with antibody against CTGF/CCN2. $\beta$-actin was used as a loading control; (B) AML12 and BRL cells were treated with increasing doses of TC $(0,10,25,50,100$ and $200 \mu \mathrm{M})$ for 12 hours. Lysates were then subjected to Western blot analysis with antibody against CTGF/CCN2. GAPDH was used as a loading control; (C) AML12 and BRL cells were treated with TC $(100 \mu \mathrm{M})$ for 6 hours before harvested, mRNA levels of CTGF/CCN2 were detected by real-time PCR. Relative mRNA levels were normalized to GAPDH and represented as mean \pm S.E.M. $(\mathrm{n}=3)$. **, $\mathrm{p}<0.01{ }^{* * *}, \mathrm{p}<0.001$; (D) AML12 and BRL cells were treated with TC $(100 \mu \mathrm{M})$ and cycloheximide $(5 \mu \mathrm{M})$ as indicated for 12 hours. Lysates were subjected to Western blot analysis with antibody against CTGF/CCN2. GAPDH was used as a loading control.

ERK, p38 MAPK, Akt, and YAP signaling are activated by TC in hepatocytes

In addition to the role in digestion, BAs were recently found to be important regulatory molecules by activating multiple signaling pathways. To explore signaling cascades related to TC-induced expression of CTGF/CCN2 in hepatocytes, we examined the activation of pathways that have been reported to mediate CTGF/CCN2 expression. Previous studies indicated that MAPK signaling is involved in regulating CTGF/CCN2 expression [27, 33, $34]$ and conjugated BAs have been reported to activate ERK and Akt signaling [20,35], we therefore examined the activation of ERK, JNK, p38 MAPK and Akt signaling pathways using Western blotting. As shown in Fig. 3A, B, TC activated ERK, Akt, p38 MAPK signaling, indicated by the increased phosphorylation of ERK, Akt, and p38 MAPK, whereas JNK signaling was not activated by TC in hepatocytes. In addition, previous studies reported that CTGF/CCN2 is a classical downstream target of Yes-associated protein (YAP) signaling [36, 37], thus, we investigated the effect of TC on YAP expression and found that YAP was increased at both the translational and transcriptional levels (Fig. 3C-E). Given that CTGF/CCN2 is mainly induced by TGF- $\beta$ in hepatocytes and TGF- $\beta$ is an important inducer in BDL-induced fibrosis [38], we investigated whether TC could activate TGF- $\beta /$ Smad signaling. Although TGF- $\beta$ mRNA level was increased in BDL rat liver (data not shown), TC stimulation failed to activate Smad 2 and Smad3 in vitro and qRT-PCR showed that TC had no effect on the mRNA level of TGF- $\beta$ (Fig. $3 \mathrm{~F}-\mathrm{H})$. Taken together, these results suggest that TC activated ERK, Akt, p38 MAPK, and YAP but not JNK and TGF- $\beta /$ Smad signaling in hepatocytes.

TC activated ERK and YAP signaling contributed to CTGF/CCN2 induction in hepatocytes

The above results show that ERK, p38 MAPK, Akt signaling, and YAP were activated by TC in AML12 and BRL hepatocytes. To further investigate whether these activated signaling cascades were involved in the induction of CTGF/CCN2, we used the MEK1/2 inhibitor (U0126), YAP function inhibitor (VP), p38 kinase inhibitor (SB203580), and Akt inhibitor (MK2206) in combination with TC to treat AML12 and BRL cells. Western blot analysis showed that the activation of these signaling pathways was inhibited by the corresponding 


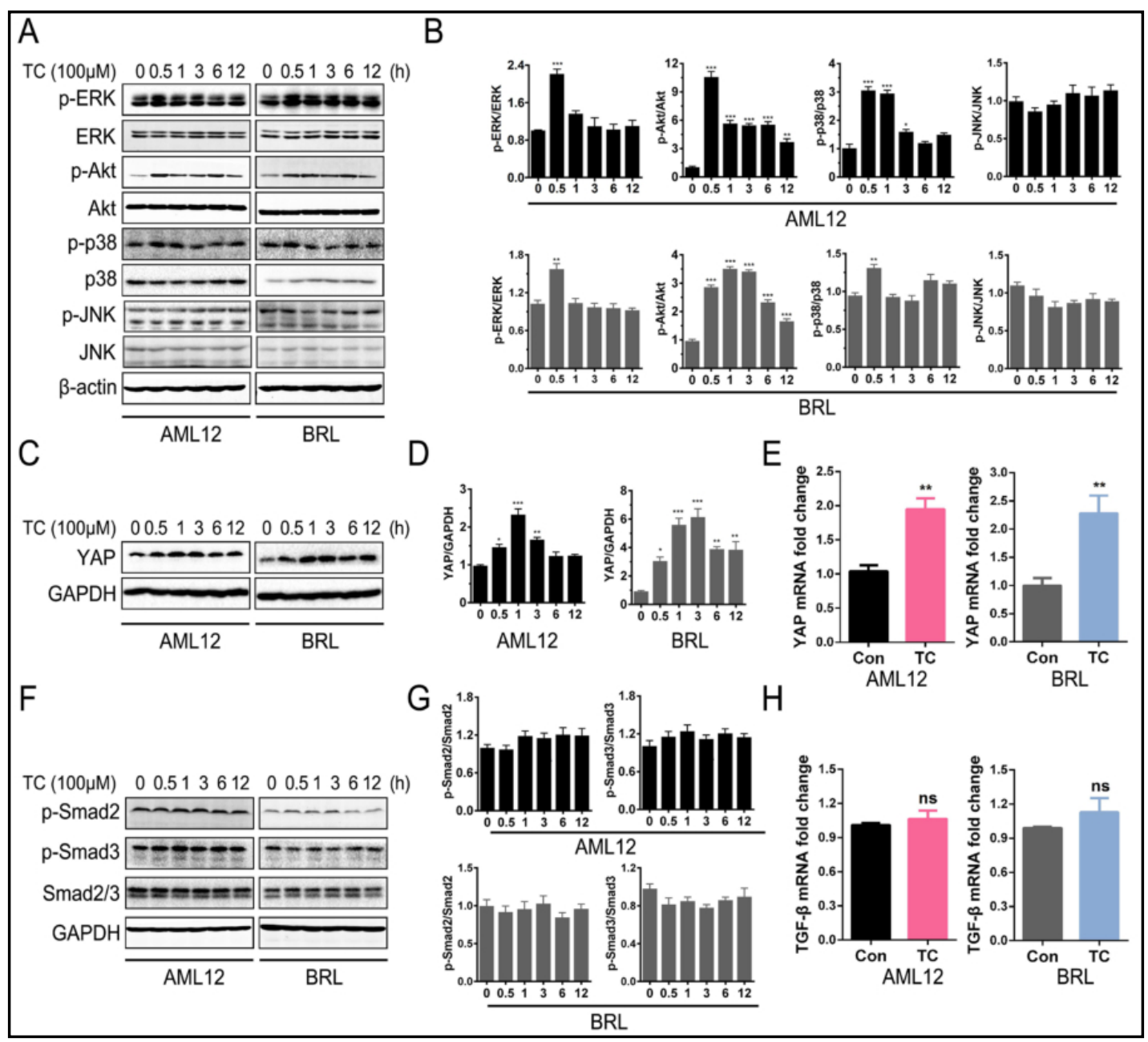

Fig. 3. ERK, Akt, p38, YAP but not JNK and TGF- $\beta /$ Smad signaling were activated by TC in hepatocytes. (A, B) AML12 and BRL cells were treated with TC $(100 \mu \mathrm{M})$ for the indicated times. Western blot was used to detect the activation of core components of MAPK and Akt signaling with antibodies against indicated proteins. $\beta$-actin was used as a loading control. Statistical analysis of relative optical density of each band was shown $(\mathrm{n}=3) .{ }^{*} \mathrm{p}<0.05{ }^{* *} \mathrm{p}<0.01 ;{ }^{* * *} \mathrm{p}<0.001$, compared with column 1 of respective chart. (C-E) The effect of TC on YAP was evaluated by Western blot and qRT-PCR, GAPDH was used as a loading control. Statistical analysis of relative optical density of each band was shown $(n=3)$. ${ }^{*} \mathrm{p}<0.05 ;{ }^{* *} \mathrm{p}<0.01 ;{ }^{* * *} \mathrm{p}<0.001$, compared with column 1 of respective chart. Relative mRNA levels were normalized to GAPDH and represented as mean \pm S.E.M. $(\mathrm{n}=3)$. ${ }^{* *}, \mathrm{p}<0.01 ;(\mathrm{F}-\mathrm{G})$ After treated with $\mathrm{TC}$ for indicated times, Western blot was used to determine the effect of TC on TGF- $\beta$ /Smad signaling, with antibodies against phospho-Smad2, phospho-Smad3 and Smad2/3, GAPDH was used as a loading control. Statistical analysis of relative optical density of each band was shown $(n=3) .(H)$ mRNA levels of TGF- $\beta$ were detected by real-time PCR after treated with TC for 6 hours. Relative mRNA levels were normalized to GAPDH and represented as mean \pm S.E.M. (n=3). ns, no significant change.

inhibitors (Fig. 4A-D). However, Western blot analysis and luciferase reporter assay of the CTGF/CCN2 promoter showed that TC-induced CTGF/CCN2 expression was abolished only in the presence of U0126 or VP (Fig. 4A-E). Interestingly, MK2206 even showed synergistic effects with TC on CTGF/CCN2 induction in hepatocytes (Fig. 4D, E). Furthermore, we used specific small interfering siRNAs targeting mek1, erk1, erk2, and yap in AML-12 and BRL cells to further confirm that TC-activated ERK and YAP signaling contributed to CTGF/CCN2 induction in hepatocytes. The results showed that siRNA directed against mek1, erk1, erk2, and yap reduced the expression of the respective targeting genes effectively (Fig. 4F-H). 
Fig. 4. TC-activated ERK, YAP but not Akt, p38 MAPK signaling contributed to CTGF/ CCN2 expression in hepatocytes. (AD) AML12 and BRL cells were treated with TC $(100 \mu \mathrm{M})$ and indicated c o n c en tration of inhibitors respectively and lysates were subjected to Western blot analyses with antibodies against indicated proteins. $\beta$-actin was used as a loading control; (E) AML12 and BRL cells were co-transfected with pRL-TK and C T G F / C C N 2 - l u c plasmids and treated with TC $(100 \mu \mathrm{M})$ and indicated con centration of inhibitors respectively for 16 h. Luciferase activity was normalized to renilla luciferase activity and expressed as the means \pm SEM of triplicate measurements. (the bars were compared as follows: $\mathrm{a}, \mathrm{p}<0.05$, compared with first; b, p <0.01, compared with first; $c, p<0.05$, compared with second; d, p <0.01, compared with second; (F) AML12 and BRL cells were transfected with specific siRNAs against erk1, erk2 (siErk1 or siErk2 cells) or co-transfected with erk1 and erk2 siRNA (siErk cells) or scramble shRNA (siSc cells) and transfected cells were treated with or without TC (100 $\mu \mathrm{M})$ for 12 hours. Lysates were subjected to Western blot analyses with antibodies against indicated proteins. $\beta$-actin was used as a loading control; (G) AML12 and BRL cells were transfected with specific siRNAs against mek1 (siMEK1 cells) or scramble shRNA (siSc cells) and transfected cells were treated with or without TC $(100 \mu \mathrm{M})$ for 12 hours. Lysates were subjected to Western blot analyses with antibodies against indicated proteins. $\beta$-actin was used as a loading control; (H) AML12 and BRL cells were transfected with specific siRNAs against yap (siYAP cells) or scramble shRNA (siSc cells) and transfected cells were treated with or without TC $(100 \mu \mathrm{M})$ for 12 hours. Lysates were subjected to Western blot analyses with antibodies against indicated proteins. $\beta$-actin was used as a loading control.

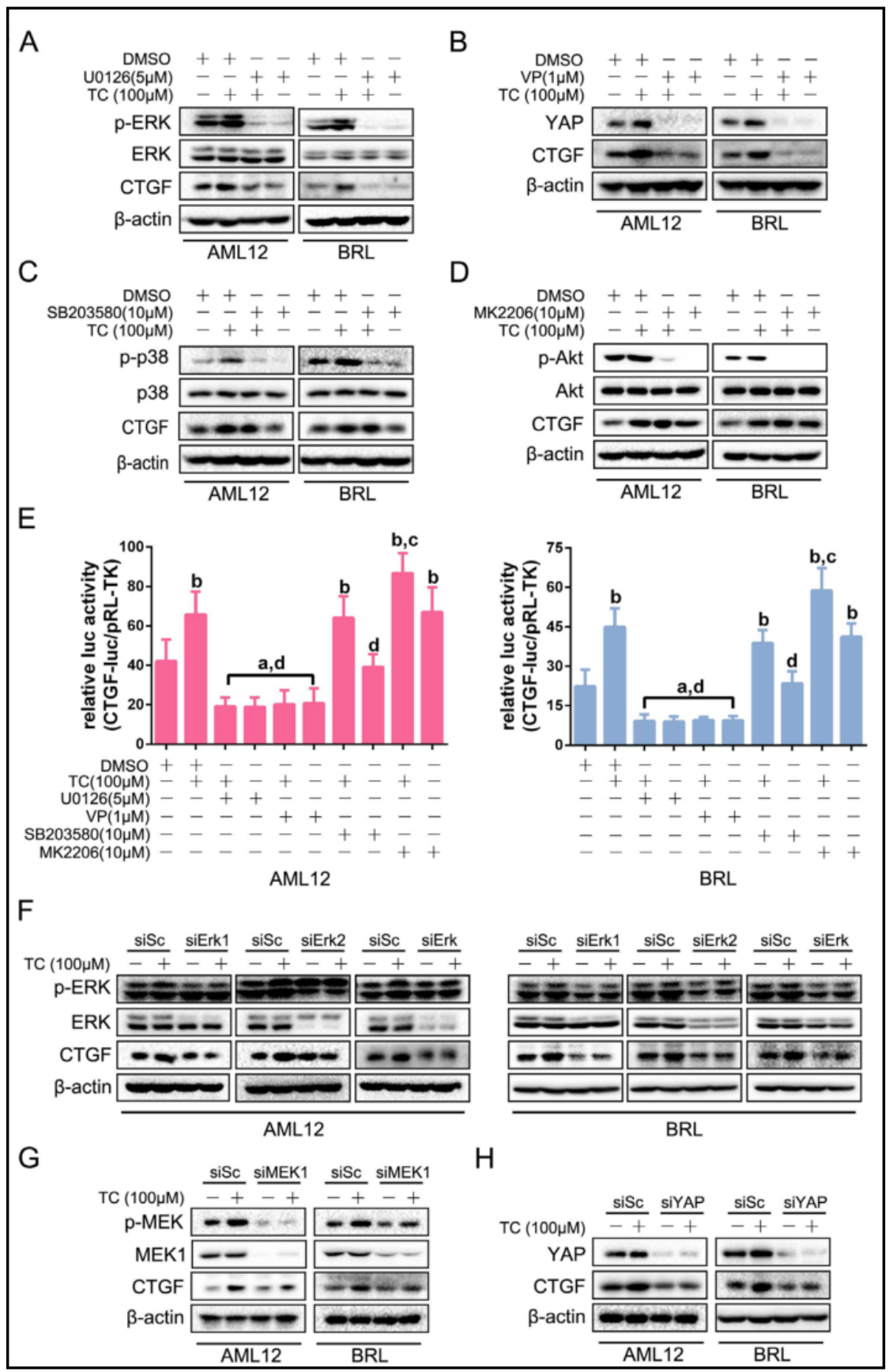




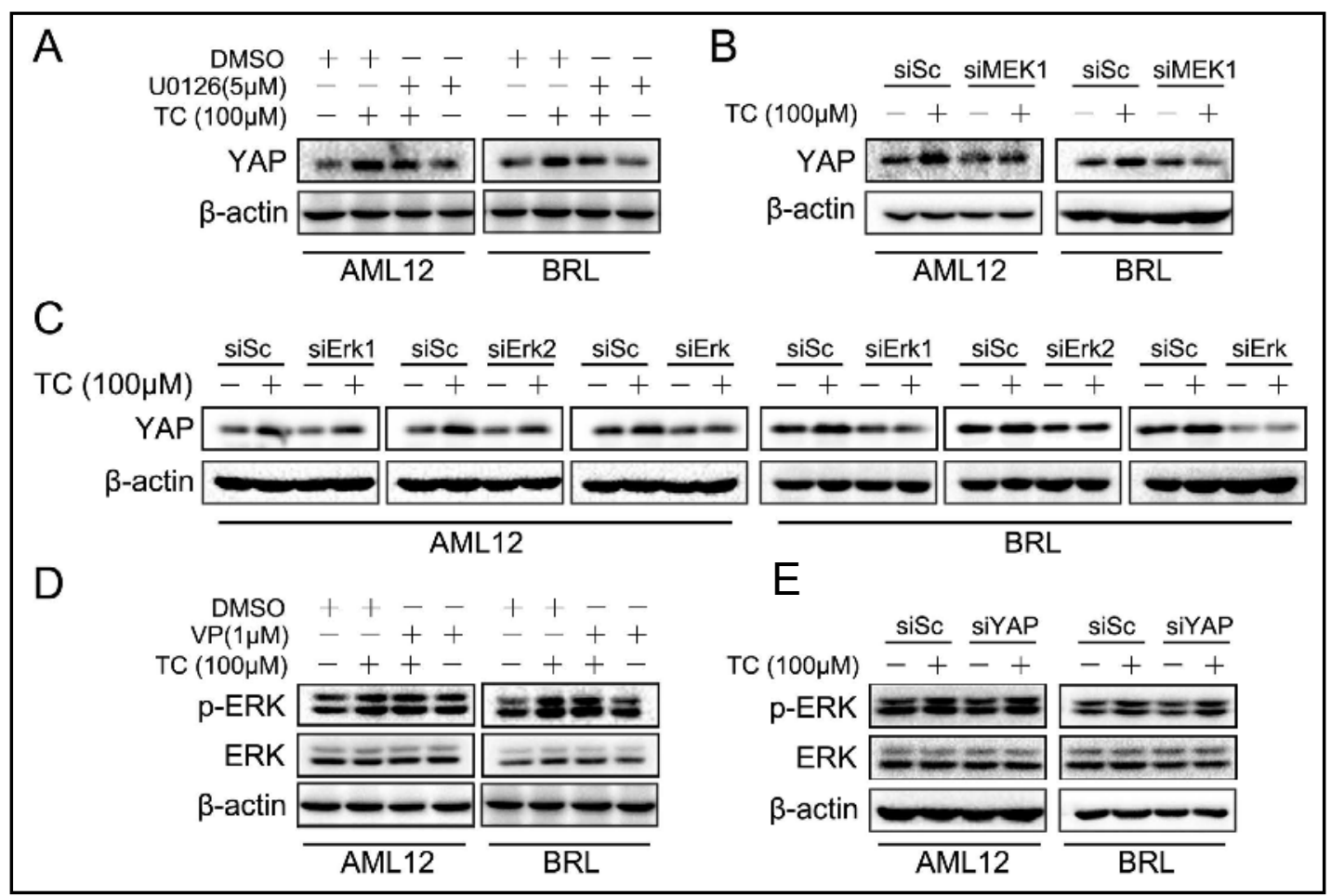

Fig. 5. YAP is a downstream molecule of TC-ERK signaling in hepatocytes.(A) AML12 and BRL cells were treated with TC $(100 \mu \mathrm{M})$ and $\mathrm{U} 0126(5 \mu \mathrm{M})$ as indicated for 12 hours, and then lysates were subjected to Western blot analysis with antibody against YAP. $\beta$-actin was used as a loading control;(B) AML12 and BRL cells were transfected with specific siRNAs against mek1 (siMEK1 cells) or scramble shRNA (siSc cells) and transfected cells were treated with or without TC $(100 \mu \mathrm{M})$ for 12 hours. Lysates were subjected to Western blot analysis with antibody against YAP. $\beta$-actin was used as a loading control; (C) AML12 and BRL cells were transfected with specific siRNAs against erk1, erk2 (siErk1 or siERK2 cells) or co-transfected with erk1 and erk2 siRNA (siERK cells) or scramble shRNA (siSc cells) and transfected cells were treated with or without TC $(100 \mu \mathrm{M})$ for 12 hours. Lysates were subjected to Western blot analysis with antibody against YAP. $\beta$-actin was used as a loading control;(D) AML12 and BRL cells were treated with TC (100 $\mu$ M) and VP $(1 \mu \mathrm{M})$ as indicated for 12 hours, and then lysates were subjected to Western blot analyses with antibodies against indicated proteins. $\beta$-actin was used as a loading control:(E) AML12 and BRL cells were transfected with specific siRNAs against yap (siYAP cells) or scramble shRNA (siSc cells) and transfected cells were treated with or without TC $(100 \mu \mathrm{M})$ for 12 hours. Lysates were subjected to Western blot analyses with antibodies against indicated proteins. $\beta$-actin was used as a loading control.

In addition, knock down of MEK1, ERK1 and 2, and YAP by siRNA attenuated CTGF/CCN2 induction by TC in both AML12 and BRL cells (Fig. 4F-H). Thus, ERK and YAP signaling contribute to TC-stimulated CTGF/CCN2 expression in hepatocytes.

\section{YAP is a downstream molecule of TC-ERK signaling in hepatocytes}

The relationship between YAP and ERK signaling is ambiguous, and previous studies reported that YAP activated ERK signaling [39-41] and that ERK inhibition downregulated the expression and activation of YAP [42]. Thus, we examined interactions between YAP and ERK signaling in TC-induced CTGF/CCN2 in hepatocytes. The MEK1/2 inhibitor U0126 inhibited TC-induced expression of YAP in both AML12 and BRL cells (Fig. 5A). Knockdown of mek1, erk1 and 2 by specific siRNAs in AML12 and BRL cells also attenuated TC-induced expression of YAP (Fig. 5B, C). However, both YAP inhibitor and specific siRNA targeting yap had no effect on the activation of TC-MEK/ERK signaling, as indicated by the protein level of phospho-ERK (Fig. 5D, E). These results indicate that YAP functions as a downstream molecule of ERK signaling in TC-induced expression of CTGF/CCN2. 


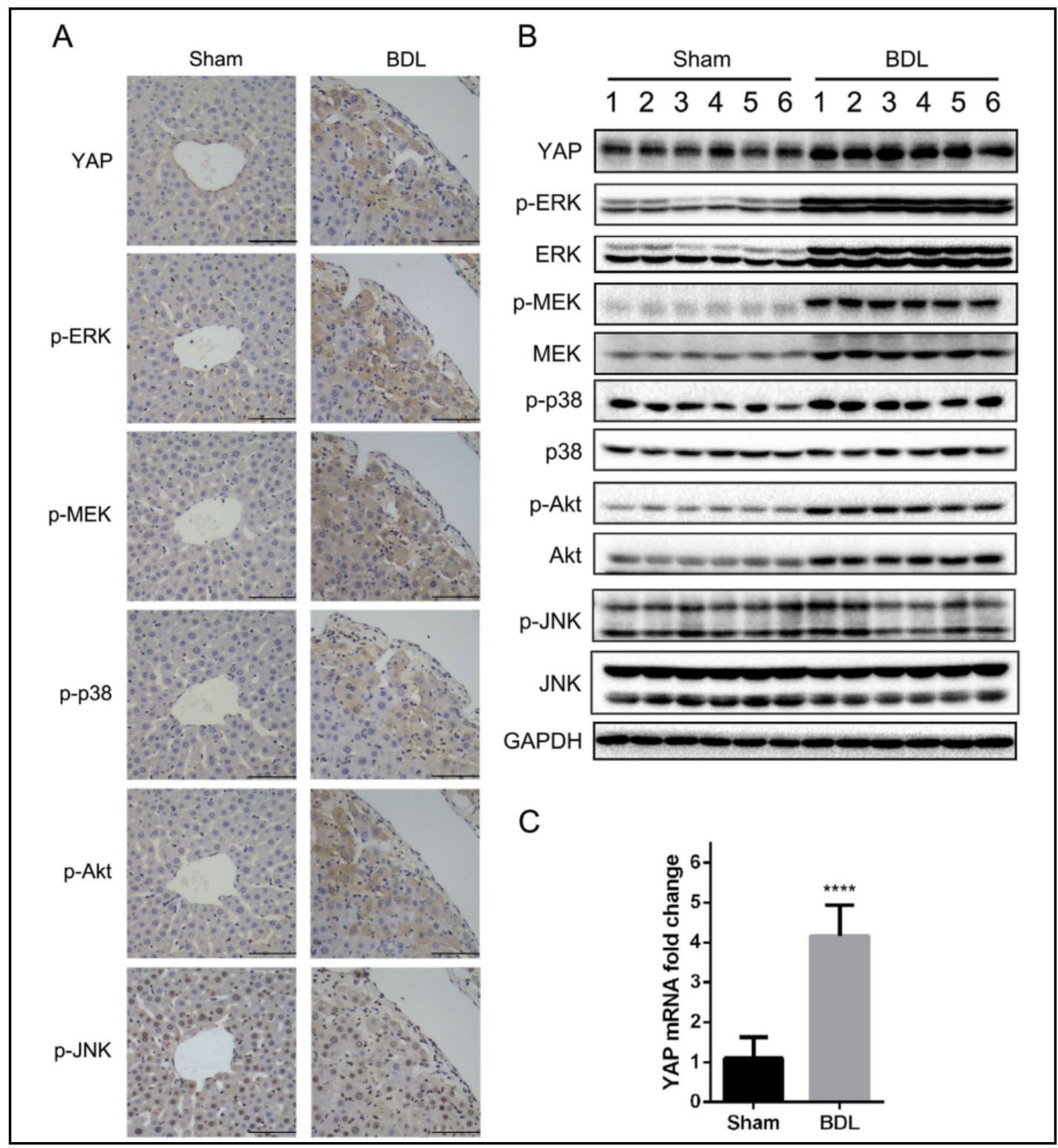

Fig. 6. MEK, ERK and YAP were enhanced expressed in cholestasis livers. (A) Serial sections of BDL and sham rats liver samples were subjected to immunohistochemical analyses of YAP, p-ERK, p-MEK, p-p38, p-Akt and p-JNK. Representative images were taken, scale bar, $100 \mu \mathrm{m}$; (B) Liver tissues of BDL and sham rats lysates were subjected to Western blot analyses with antibodies against indicated proteins. GAPDH was used as a loading control; (C) mRNA levels of YAP of liver tissues from BDL and sham rats were detected by real-time PCR. Relative mRNA levels were normalized to GAPDH and represented as mean \pm S.E.M. $(n=3)$. $* * * *, \mathrm{p}<0.0001$.

The expression of YAP and the activity of MEK/ERK, p38, Akt signaling were all elevated in the cholestatic liver

We then measured the expression of YAP and activation of MEK/ERK, p38, Akt, and JNK signaling in BDL rat liver. Analyses of serial immunostained liver sections showed that YAP, phospho-MEK, phospho-ERK, phospho-p38, and phospho-Akt were all increased and partly co-expressed in the BDL-induced cholestatic liver (Fig. 6A). But phospho-JNK was not elevated in the BDL-induced cholestatic liver, which was consistent with the in vitro results. Additionally, Western blotting showed enhanced expression of YAP, p-MEK, p-ERK, p-p38, p-Akt but not p-JNK in BDL rat liver tissue compared with that in the sham group (Fig. 6B). 




Fig. 7. TC-Akt signaling antagonize TC-MEK/ERK signaling in hepatocytes. (A) AML12 and BRL cells were treated with TC $(100 \mu \mathrm{M})$ and MK2206 $(10 \mu \mathrm{M})$ as indicated for 12 hours, and then lysates were subjected to Western blot analyses with antibodies against indicated proteins. GAPDH was used as a loading control; (B) AML12 and BRL cells were treated with TC $(100 \mu \mathrm{M})$ and U0126 $(5 \mu \mathrm{M})$ as indicated for 12 hours, then Western blot was used to check the activation of Akt signaling as indicated by the phospho-Akt; GAPDH was used as a loading control; (C) AML12 and BRL cells were treated with TC $(100 \mu \mathrm{M})$ and VP $(1 \mu \mathrm{M})$ as indicated for 12 hours, then Western blot was used to check the activation of Akt signaling as indicated by the phospho-Akt; GAPDH was used as a loading control; (D) Schematic illustration of TC-induced CTGF/ CCN2 in hepatocytes.

Real-time PCR analysis showed that YAP mRNA level of the BDL rat liver was higher than that of the sham group (Fig. 6C). Taken together, these results prove that YAP expression and MEK-ERK, p38, Akt signaling activation are all elevated in cholestatic livers.

\section{TC-Akt signaling antagonize TC-MEK/ERK signaling in hepatocytes}

Of note, we observed that inhibition of Akt signaling increased the level of CTGF/CCN2 in the presence or absence of TC stimulation in hepatocytes. To further illustrate this finding, we tested the activation of ERK signaling and the level of YAP in AML12 and BRL cells treated with MK2206. Inhibiting Akt signaling with MK2206 resulted in elevated TC-activated ERK and downstream YAP signaling in both AML12 and BRL cells (Fig. 7A). On the contrary, both MEK1/2 inhibitor and YAP inhibitor showed no effect on activation of TC-Akt signaling (Fig. 7B, C). Collectively, these results suggest that TC-Akt signaling shows an antagonistic effect on TC-ERK signaling in regulating the expression of CTGF/CCN2 in hepatocytes (Fig. 7D).

\section{Discussion}

BAs, the major constituents of bile, are synthesized from cholesterol in the liver and secreted into the intestine to facilitate absorption [6]. Impairment of bile formation or interruption of bile flow can lead to cholestasis [43]. During cholestasis, the high level of toxic BAs cause apoptosis and necrosis of hepatocytes, activate inflammatory signaling cascades, and promote proliferation of HSCs [15-18], all of which contribute to liver fibrosis. 


\section{Cellular Physiology Cell Physiol Biochem 2018;50:1711-1725

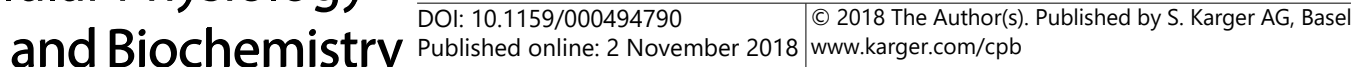 \\ Yu et al.: Taurocholate Induces CTGF in Hepatocytes}

Recent investigations revealed that BAs function as regulators of several cellular signaling pathways like JNK, ERK, and Akt signaling [19-21]. The precise roles of signaling cascades activated by BAs are worth to be explored.

In this study, we used a BDL rat model, a classic experimental model of cholestasis, to induce obstructive cholestatic injury in rats [44] and found that the classical fibrotic matricellular protein CTGF/CCN2 was dramatically increased in BDL-induced liver fibrosis. CTGF/CCN2 is a fibrogenic master switch in the fibrotic liver diseases, and is synthesized and secreted by various types of cells in the liver [24]; CTGF/CCN2 inhibition has been shown to prevent liver fibrosis [45]. Extracellular stimuli like EGF and TGF- $\beta$ [26, 37] regulate CTGF/ CCN2 expression through different intracellular signaling pathways. Besides, in cholestasisinduced human cirrhosis, CTGF/CCN2 expression was also found to be increased [31]. Thus, we wondered whether BA can act as an inducer of CTGF/CCN2 through certain signaling pathways. To investigate this assumption, we used the hepatic cell lines, AML12 and BRL, to explore the effect of BAs on CTGF/CCN2 expression. We found that TC, one of the major substantially elevated BAs in the cholestatic liver $[12,13]$, upregulated CTGF/CCN2 levels in both AML12 and BRL cells. Other groups have reported that JNK, Akt, and ERK were activated by BAs and our previous study indicated that ERK, JNK, and p38 MAPK signaling mediated CTGF/CCN2 expression in liver progenitor cells [27], and so we checked whether TC could activate these pathways in hepatocytes. Our results showed that TC activated ERK, p38 MAPK, and Akt, but not JNK signaling cascades. Consistent with previous reports, JNK signaling was not activated in hepatocytes in BDL-induced cholestasis [46]. Furthermore, YAP was reported to be involved in cholestasis-related liver fibrosis and liver cancer progression, and served as a classical upstream regulator of CTGF/CCN2 in mammalian cells [29, 30]. Thus, we investigated whether BAs regulate CTGF/CCN2 levels through YAP and found that TC increased YAP levels in hepatocytes. Moreover, when combined with respective inhibitors, we observed that only ERK and YAP activation mediated TC-induced production of CTGF/ CCN2. Although TGF- $\beta$ was reported to be a main inducer of CTGF/CCN2 in hepatocytes [47], we found that TC neither activated Smad2/Smad3 nor enhanced the synthesis of TGF- $\beta$, which indicated that TGF- $\beta /$ Smad signaling was not involved in TC-induced CTGF/CCN2 in hepatocytes.

The relationship between ERK signaling and YAP was elusive; YAP expression levels affected ERK1/2 phosphorylation in human hepatocellular carcinoma, cutaneous squamous cell carcinoma, and breast cancer cell lines [39-41], while in non-small cell lung cancer cells, reducing the ERK levels could decrease YAP expression [42]. In our study, we observed that MEK/ERK inhibitors U0126 and specific siRNAs targeting mek1, erk1, and erk2 decreased TC-induced expression of YAP and CTGF/CCN2 in hepatocytes. In contrast, inhibitor of YAP as well as siRNAs targeting yap caused the reduction in the expression of CTGF/CCN2, but showed no effect on activation of ERK signaling in hepatocytes. Consistent with the in vitro results, p-MEK, p-ERK, and YAP levels were all upregulated in BDL rat liver compared to the sham group. Taken together, these results suggest that ERK signaling serves as an upstream regulator of YAP in CTGF/CCN2 expression in hepatocytes.

Extracellular signals like EGF and IGF were found to activate both ERK and Akt signaling, whereas the relationship between Akt and ERK signaling was not clear in hepatocytes. In addition, the role of Akt signaling in fibrosis is controversial and dependent on cell context; IL-10 apparently inhibited fibrosis by activating Akt signaling [48], whereas in hepatic stellate cells, Akt signaling showed profibrotic effects [49]. Here, we observed that TC activated both ERK and Akt signaling, and Akt signaling inhibited TC-activated ERK signaling in hepatocytes, suggesting that the TC-ERK/YAP/CTGF/CCN2 axis may be inhibited by the TC-Akt axis in hepatocytes. Therefore, Akt signaling may exert anti-fibrotic effects in hepatocytes during cholestasis and may be a promising therapeutic target for cholestatic diseases, but the comprehensive effect of Akt inhibitors needs to be further explored in the liver. 


\section{Cellular Physiology Cell Physiol Biochem 2018;50:1711-1725 \begin{tabular}{ll|l} 
and Biochemistry Published online: 2 November 2018 & $\begin{array}{l}\text { (c) } 2018 \text { The Author(s). Published by S. Karger AG, Basel } \\
\text { www.karger.com/cpb }\end{array}$ \\
\hline
\end{tabular} \\ Yu et al.: Taurocholate Induces CTGF in Hepatocytes}

\section{Conclusion}

In this study, we confirmed that CTGF/CCN2 was increased in hepatocytes during cholestasis-induced liver fibrosis. Additionally, TC mediated the de novo synthesis of CTGF/CCN2 in hepatocytes. Furthermore, TC-activated ERK-YAP axis contributed to the upregulation of CTGF/CCN2 in hepatocytes, whereas TC-activated Akt signaling showed an inhibitory effect on the ERK-YAP axis and the expression of CTGF/CCN2. These results have provided evidence for the fibrotic role of TC in the liver, suggesting that elevated TC and its downstream ERK-YAP signaling may be potential therapeutic targets in cholestatic liver fibrosis (Fig. 7D).

\section{Abbreviations}

BA (bile acid); BDL (bile duct ligation); CTGF/CCN2 (connective tissue growth factor); ERK (extracellular signal-regulated kinase); GAPDH (glyceraldehyde-3-phosphate dehydrogenase); HSCs (hepatic stellate cells); MAPK (mitogen-activated protein kinase); siRNA (small interfering RNA); TC (taurocholate); TGF- $\beta$ (transforming growth factor beta); VP (Verteporfin); YAP (Yes-associated protein).

\section{Acknowledgements}

We are grateful to Prof. Jian-Guo Song (SIBS, Chinese Academy of Sciences) for providing the AML12 cell line. We also thank CureEdit Bioscience Ltd. (Houston, USA) for their assistance in editing this manuscript. This work was supported by the National Nature Science Foundation of China (grant numbers 81401997 and 81500565). BY performed experiments, analyzed the data, designed the figures, and drafted the manuscript.

GJ and MM were also involved in performing experiments. BZ, and XC assisted with analyzing and interpreting data, and provided technical support. ZD designed the research, analyzed the data, critical revision of manuscript for important intellectual content, and obtained funding.

\section{Disclosure Statement}

The authors declare that the research was conducted in the absence of any commercial or financial relationships that could be construed as a potential conflict of interest.

\section{References}

1 Trauner M, Meier PJ, Boyer JL: Molecular pathogenesis of cholestasis. N Engl J Med 1998;339:1217-1227.

-2 Webb GJ, Hirschfield GM: Primary biliary cholangitis in 2016: High-definition PBC: biology, models and therapeutic advances. Nat Rev Gastroenterol Hepatol 2017;14:76-78.

- Eaton JE, Talwalkar JA, Lazaridis KN, Gores GJ, Lindor KD: Pathogenesis of primary sclerosing cholangitis and advances in diagnosis and management. Gastroenterology 2013;145:521-536.

4 Asai A, Miethke A, Bezerra JA: Pathogenesis of biliary atresia: defining biology to understand clinical phenotypes. Nat Rev Gastroenterol Hepatol 2015;12:342-352.

5 de Vries E, Beuers U: Management of cholestatic disease in 2017. Liver Int 2017;37 Suppl 1:123-129.

-6 Russell DW: The enzymes, regulation, and genetics of bile acid synthesis. Annu Rev Biochem 2003;72:137174.

7 Chiang JY: Regulation of bile acid synthesis: pathways, nuclear receptors, and mechanisms. J Hepatol 2004;40:539-551. 


\section{Cellular Physiology Cell Physiol Biochem 2018;50:1711-1725 \begin{tabular}{l|l|l} 
and Biochemistry Published online: 2 November 2018 & $\begin{array}{l}\odot 2018 \text { The Author(s). Published by S. Karger AG, Basel } \\
\text { www.karger.com/cpb }\end{array}$ \\
\hline
\end{tabular} \\ Yu et al.: Taurocholate Induces CTGF in Hepatocytes}

-8 Schaap FG, van der Gaag NA, Gouma DJ, Jansen PL: High expression of the bile salt-homeostatic hormone fibroblast growth factor 19 in the liver of patients with extrahepatic cholestasis. Hepatology 2009;49:12281235.

-9 Gartung C, Ananthanarayanan M, Rahman MA, Schuele S, Nundy S, Soroka CJ, Stolz A, Suchy FJ, Boyer JL: Down-regulation of expression and function of the rat liver $\mathrm{Na}+$ /bile acid cotransporter in extrahepatic cholestasis. Gastroenterology 1996;110:199-209.

10 Sauer P, Stiehl A, Fitscher BA, Riedel HD, Benz C, Kloters-Plachky P, Stengelin S, Stremmel W, Kramer W: Downregulation of ileal bile acid absorption in bile-duct-ligated rats. J Hepatol 2000;33:2-8.

$\checkmark 11$ Setchell KD, Rodrigues CM, Clerici C, Solinas A, Morelli A, Gartung C, Boyer J: Bile acid concentrations in human and rat liver tissue and in hepatocyte nuclei. Gastroenterology 1997;112:226-235.

12 Zhang Y, Hong JY, Rockwell CE, Copple BL, Jaeschke H, Klaassen CD: Effect of bile duct ligation on bile acid composition in mouse serum and liver. Liver Int 2012;32:58-69.

13 Zhou K, Lin N, Xiao Y, Wang Y, Wen J, Zou GM, Gu X, Cai W: Elevated bile acids in newborns with Biliary Atresia (BA). PLoS One 2012;7:e49270.

14 Amaral JD, Viana RJ, Ramalho RM, Steer CJ, Rodrigues CM: Bile acids: regulation of apoptosis by ursodeoxycholic acid. J Lipid Res 2009;50:1721-1734.

15 Allen K, Jaeschke H, Copple BL: Bile acids induce inflammatory genes in hepatocytes: a novel mechanism of inflammation during obstructive cholestasis. Am J Pathol 2011;178:175-186.

16 Yerushalmi B, Dahl R, Devereaux MW, Gumpricht E, Sokol RJ: Bile acid-induced rat hepatocyte apoptosis is inhibited by antioxidants and blockers of the mitochondrial permeability transition. Hepatology 2001;33:616-626.

17 Malhi H, Guicciardi ME, Gores GJ: Hepatocyte death: a clear and present danger. Physiol Rev 2010;90:11651194.

-18 Svegliati-Baroni G, Ridolfi F, Hannivoort R, Saccomanno S, Homan M, De Minicis S, Jansen PL, Candelaresi C, Benedetti A, Moshage H: Bile acids induce hepatic stellate cell proliferation via activation of the epidermal growth factor receptor. Gastroenterology 2005;128:1042-1055.

19 Gupta S, Stravitz RT, Dent P, Hylemon PB: Down-regulation of cholesterol 7alpha-hydroxylase (CYP7A1) gene expression by bile acids in primary rat hepatocytes is mediated by the c-Jun N-terminal kinase pathway. J Biol Chem 2001;276:15816-15822.

20 Cao R, Cronk ZX, Zha W, Sun L, Wang X, Fang Y, Studer E, Zhou H, Pandak WM, Dent P, Gil G, Hylemon PB: Bile acids regulate hepatic gluconeogenic genes and farnesoid $X$ receptor via G(alpha)i-protein-coupled receptors and the AKT pathway. J Lipid Res 2010;51:2234-2244.

21 Dent P, Fang Y, Gupta S, Studer E, Mitchell C, Spiegel S, Hylemon PB: Conjugated bile acids promote ERK1/2 and AKT activation via a pertussis toxin-sensitive mechanism in murine and human hepatocytes. Hepatology 2005;42:1291-1299.

22 Holbourn KP, Acharya KR, Perbal B: The CCN family of proteins: structure-function relationships. Trends Biochem Sci 2008;33:461-473.

23 Luft FC: CCN2, the connective tissue growth factor. J Mol Med (Berl) 2008;86:1-3.

-24 Gressner OA, Gressner AM: Connective tissue growth factor: a fibrogenic master switch in fibrotic liver diseases. Liver Int 2008;28:1065-1079.

-25 Gressner OA, Weiskirchen R, Gressner AM: Biomarkers of liver fibrosis: clinical translation of molecular pathogenesis or based on liver-dependent malfunction tests. Clin Chim Acta 2007;381:107-113.

26 Gressner OA, Lahme B, Siluschek M, Rehbein K, Weiskirchen R, Gressner AM: Connective tissue growth factor is a Smad2 regulated amplifier of transforming growth factor beta actions in hepatocytes--but without modulating bone morphogenetic protein 7 signaling. Hepatology 2009;49:2021-2030.

-27 Ding ZY, Jin GN, Liang HF, Wang W, Chen WX, Datta PK, Zhang MZ, Zhang B, Chen XP: Transforming growth factor beta induces expression of connective tissue growth factor in hepatic progenitor cells through Smad independent signaling. Cell Signal 2013;25:1981-1992.

28 Zhao B, Wei X, Li W, Udan RS, Yang Q Kim J, Xie J, Ikenoue T, Yu J, Li L, Zheng P, Ye K, Chinnaiyan A, Halder G, Lai ZC, Guan KL: Inactivation of YAP oncoprotein by the Hippo pathway is involved in cell contact inhibition and tissue growth control. Genes Dev 2007;21:2747-2761.

29 Bai H, Zhang N, Xu Y, Chen Q, Khan M, Potter JJ, Nayar SK, Cornish T, Alpini G, Bronk S, Pan D, Anders RA: Yes-associated protein regulates the hepatic response after bile duct ligation. Hepatology 2012;56:10971107. 


\section{Cellular Physiology Cell Physiol Biochem 2018;50:1711-1725 \begin{tabular}{l|l|l|l} 
DOI: 10.1159/000494790 & ○ 2018 The Author(s). Published by S. Karger AG, Basel
\end{tabular} and Biochemistry Published online: 2 November 2018 www.karger.com/cpb}

Yu et al.: Taurocholate Induces CTGF in Hepatocytes

30 Gurda GT, Zhu Q, Bai H, Pan D, Schwarz KB, Anders RA: The use of Yes-associated protein expression in the diagnosis of persistent neonatal cholestatic liver disease. Hum Pathol 2014;45:1057-1064.

-31 Williams EJ, Gaca MD, Brigstock DR, Arthur MJ, Benyon RC: Increased expression of connective tissue growth factor in fibrotic human liver and in activated hepatic stellate cells. J Hepatol 2000;32:754-761.

32 Pan X, Wang X, Lei W, Min L, Yang Y, Wang X, Song J: Nitric oxide suppresses transforming growth factorbeta1-induced epithelial-to-mesenchymal transition and apoptosis in mouse hepatocytes. Hepatology 2009;50:1577-1587.

-33 Meyer C, Godoy P, Bachmann A, Liu Y, Barzan D, Ilkavets I, Maier P, Herskind C, Hengstler JG, Dooley S: Distinct role of endocytosis for Smad and non-Smad TGF-beta signaling regulation in hepatocytes. J Hepatol 2011;55:369-378.

-34 Seo HY, Jung YA, Lee SH, Hwang JS, Park KG, Kim MK, Jang BK: Kahweol decreases hepatic fibrosis by inhibiting the expression of connective tissue growth factor via the transforming growth factor-beta signaling pathway. Oncotarget 2017;8:87086-87094.

-35 Rao YP, Studer EJ, Stravitz RT, Gupta S, Qiao L, Dent P, Hylemon PB: Activation of the Raf-1/MEK/ERK cascade by bile acids occurs via the epidermal growth factor receptor in primary rat hepatocytes. Hepatology 2002;35:307-314.

-36 Quan T, Xu Y, Qin Z, Robichaud P, Betcher S, Calderone K, He T, Johnson TM, Voorhees JJ, Fisher GJ: Elevated YAP and its downstream targets CCN1 and CCN2 in basal cell carcinoma: impact on keratinocyte proliferation and stromal cell activation. Am J Pathol 2014;184:937-943.

-37 Urtasun R, Latasa MU, Demartis MI, Balzani S, Goni S, Garcia-Irigoyen O, Elizalde M, Azcona M, Pascale RM, Feo F, Bioulac-Sage P, Balabaud C, Muntane J, Prieto J, Berasain C, Avila MA: Connective tissue growth factor autocriny in human hepatocellular carcinoma: oncogenic role and regulation by epidermal growth factor receptor/yes-associated protein-mediated activation. Hepatology 2011;54:2149-2158.

38 Arias M, Sauer-Lehnen S, Treptau J, Janoschek N, Theuerkauf I, Buettner R, Gressner AM, Weiskirchen R: Adenoviral expression of a transforming growth factor-beta 1 antisense mRNA is effective in preventing liver fibrosis in bile-duct ligated rats. BMC Gastroenterol 2003;3:29.

-39 Xu MZ, Chan SW, Liu AM, Wong KF, Fan ST, Chen J, Poon RT, Zender L, Lowe SW, Hong W, Luk JM: AXL receptor kinase is a mediator of YAP-dependent oncogenic functions in hepatocellular carcinoma. Oncogene 2011;30:1229-1240.

40 Jia J, Li C, Luo S, Liu-Smith F, Yang J, Wang X, Wang N, Lai B, Lei T, Wang Q Xiao S, Shao Y, Zheng Y: YesAssociated Protein Contributes to the Development of Human Cutaneous Squamous Cell Carcinoma via Activation of RAS. J Invest Dermatol 2016;136:1267-1277.

41 Overholtzer M, Zhang J, Smolen GA, Muir B, Li W, Sgroi DC, Deng CX, Brugge JS, Haber DA: Transforming properties of YAP, a candidate oncogene on the chromosome 11q22 amplicon. Proc Natl Acad Sci U S A 2006;103:12405-12410.

-42 You B, Yang YL, Xu Z, Dai Y, Liu S, Mao JH, Tetsu O, Li H, Jablons DM, You L: Inhibition of ERK1/2 downregulates the Hippo/YAP signaling pathway in human NSCLC cells. Oncotarget 2015;6:4357-4368.

-43 Elferink RO: Cholestasis. Gut 2003;52 Suppl 2:ii42-48.

44 Liedtke C, Luedde T, Sauerbruch T, Scholten D, Streetz K, Tacke F, Tolba R, Trautwein C, Trebicka J, Weiskirchen R: Experimental liver fibrosis research: update on animal models, legal issues and translational aspects. Fibrogenesis Tissue Repair 2013;6:19.

-45 Tong Z, Chen R, Alt DS, Kemper S, Perbal B, Brigstock DR: Susceptibility to liver fibrosis in mice expressing a connective tissue growth factor transgene in hepatocytes. Hepatology 2009;50:939-947.

46 Kluwe J, Pradere JP, Gwak GY, Mencin A, De Minicis S, Osterreicher CH, Colmenero J, Bataller R, Schwabe RF: Modulation of hepatic fibrosis by c-Jun-N-terminal kinase inhibition. Gastroenterology 2010;138:347-359.

-47 Weng HL, Ciuclan L, Liu Y, Hamzavi J, Godoy P, Gaitantzi H, Kanzler S, Heuchel R, Ueberham U, Gebhardt R, Breitkopf K, Dooley S: Profibrogenic transforming growth factor-beta/activin receptor-like kinase 5 signaling via connective tissue growth factor expression in hepatocytes. Hepatology 2007;46:1257-1270.

-48 Shi J, Li J, Guan H, Cai W, Bai X, Fang X, Hu X, Wang Y, Wang H, Zheng Z, Su L, Hu D, Zhu X: Anti-fibrotic actions of interleukin-10 against hypertrophic scarring by activation of PI3K/AKT and STAT3 signaling pathways in scar-forming fibroblasts. PLoS One 2014;9:e98228.

49 Yu DK, Zhang CX, Zhao SS, Zhang SH, Zhang H, Cai SY, Shao RG, He HW: The anti-fibrotic effects of epigallocatechin-3-gallate in bile duct-ligated cholestatic rats and human hepatic stellate LX-2 cells are mediated by the PI3K/Akt/Smad pathway. Acta Pharmacol Sin 2015;36:473-482. 